\title{
Bone morphogenetic protein 7 suppresses the progression of hepatic fibrosis and regulates the expression of gremlin and transforming growth factor $\beta 1$
}

\author{
TAO YANG*, SHAO LONG CHEN*, XIAO JU LU, CHUN YAN SHEN, YAN LIU and YONG PING CHEN \\ Department of Infection and Liver Diseases, Liver Research Center, The First Affiliated Hospital \\ of Wenzhou Medical College, Wenzhou, Zhejiang 325000, P.R. China
}

Received January 8, 2012; Accepted April 20, 2012

DOI: $10.3892 / \mathrm{mmr} .2012 .892$

\begin{abstract}
Bone morphogenetic protein 7 (BMP-7), a member of the transforming growth factor (TGF)- $\beta$ superfamily, counteracts the effect of TGF- $\beta$ through different signaling pathways, and gremlin is considered as one of the antagonists of BMP-7. The aim of this study was to investigate the antifibrotic effect of BMP-7, and to clarify the expression patterns of gremlin and TGF- $\beta 1$ in the progression of hepatic fibrosis after treatment with BMP-7. A mouse liver fibrosis model was induced by hypodermic injection of $\mathrm{CCL}_{4}$ and all the liver and blood samples were preserved for further study. Reverse transcription-polymerase chain reaction was used for detecting mRNA expression, and protein levels and localization were measured by western blotting and immunohistochemistry, respectively. The improvement of liver function and the regression of hepatic fibrosis were demonstrated by the parameters of a liver test and a histopathological assay, owing to the downregulated expression of COL-I, $\alpha$-SMA, TIMP-2 and upregulated MMP-2. Moreover, exogenous BMP-7 appeared to suppress the expression of TGF- $\beta 1$ and increase the levels of gremlin. In conclusion, hepatic fibrosis was ameliorated by the administration of BMP-7, and the expression of gremlin and TGF- $\beta 1$ were regulated by BMP-7. The identification of the dynamic expression pattern of gremlin may yield a novel biomarker for assessing the degree of hepatic fibrosis.
\end{abstract}

\section{Introduction}

Liver cirrhosis, the late stage of fibrosis, manifests a significant world health care burden arising from chronic liver injury,

Correspondence to: Dr Yong Ping Chen, Department of Infection and Liver Diseases, Liver Research Center, The First Affiliated Hospital of Wenzhou Medical College, 2 Fuxue Lane, Wenzhou, Zhejiang 325000, P.R. China

E-mail: ypchen106@yahoo.com.cn

${ }^{*}$ Contributed equally

Key words: bone morphogenetic protein 7, gremlin, hepatic fibrosis, transforming growth factor $\beta 1$ such as chronic viral hepatitis, excess alcohol consumption, the metabolic syndrome, as well as autoimmune disease, and leads to complications and mortality worldwide (1). Fibrosis represents a wound-healing response to liver injury, characterized by an excessive deposition of extracellular matrices (ECMs), such as collagens and glycoproteins (2). Among the various members of the collagen family, collagen I and III are found to mostly relate to fibrogenesis, and their expression levels are paralleled with the progression of liver fibrosis. Additionally, hyaluronic acid (HA) that belongs to the glycoprotein family, is strongly associated with the severity of fibrosis, therefore the expression patterns of HA are widely used in clinics to assess the degree of fibrosis (3). The liver can be restored to its normal function and structure when the injury is transient, self-limited or controlled by active treatment (4). However, if the primary cause of the injury is left untreated, chronic inflammation and a persistent deposit of ECM progressively lead to cirrhosis, which is characterized by the formation of regenerative nodules, diffuse fibrosis and alerted liver function (5). Continued advances in our understanding of the mechanism underlying fibrogenesis has yielded the recognition that liver fibrosis, or even cirrhosis, may be reversible, thereby making novel therapeutic strategies for the reversion of hepatic fibrosis a crucial subject for research (6).

The primary cell type that is involved in the excessive deposition of ECM are the hepatic stellate cells (HSCs), which are activated in response to chronic injury. In this well-regulated process, vitamin A-storing HSCs are converted into contractile myofibroblasts (activated HSCs), characterized by an increased expression of $\alpha$-SMA, collagen and the production of various fibrosis-related cytokines, such as transforming growth factor (TGF)- $\beta$, constructive tissue growth factor (CTGF), tissue inhibitors of matrix metalloproteinases (TIMPs), orchestrating the complex molecular regulatory system underlying fibrogenesis $(7,8)$. Among the large number of cytokines and growth factors in the progression of hepatic fibrosis, TGF- $\beta$ signaling is considered a key determinant, owing to emerging evidence indicating that TGF- $\beta$ is a critical fibrinogenic and proliferative stimulus to HSCs during fibrogenesis $(9,10)$. The TGF- $\beta$ family consists of three types of TGF isoforms ( $\beta 1, \beta 2$ and $\beta 3)$. Of these, TGF- $\beta 1$, that is mainly released by Kupffer cells in the initial process, is mostly related to fibrogenesis (11). By 
contrast, TGF- $\beta$ exerts its unique effort via the TGF- $\beta /$ Smad signaling pathway, which is implicated in various physiological and pathological conditions, such as embryogenesis, inflammation and fibrosis (12). Moreover, the balance of MMPs and their inhibitors TIMPs are also regulated by TGF- $\beta$, thereby further highlighting the crucial role of TGF- $\beta$ in the progression of fibrosis $(13,14)$.

Bone morphogenetic protein-7 (BMP-7) belongs to the TGF- $\beta$ superfamily that contains TGF- $\beta$, activins and bone morphogenetic proteins. BMPs have been studied extensively for their involvement in embryonic development and cell differentiation, whereas, recently, a role for the BMP-7 signaling pathway in the regulation of fibrosis was recognized based on the counteractive effect of BMP-7 against the TGF- $\beta /$ Smad signaling pathways (15). TGF- $\beta$ activates the smad $2 / 3$ proteins, while BMP-7 carries out its function by forming a receptor complex that subsequently promotes the phosphorylation of Smad1/5/8, leading to the suppression of the effects of TGF- $\beta(16,17)$. There is increasing awareness of the contribution of the TGF- $\beta$ signaling pathway in pathological conditions, therefore BMP-7 attracts extensive attention for its therapeutic potential by counteracting the effect of TGF- $\beta$. For example, TGF- $\beta$ is considered to be the potent factor in promoting epithelial-to-mesenchymal transition (EMT), and is recognized as an important event in fibrogenesis and carcinogenesis, whereas TGF- $\beta$-induced EMT was found to be suppressed by the administration of human recombinant BMP-7 in a rodent model of chronic renal injury, indicating a role of BMP-7 in the reversal of renal fibrosis (18). Based on the above observation, the issue remains whether BMP-7 has the same effect on liver fibrosis. Therefore, in the present study, we explored the possible antifibrotic effect of BMP-7 in a mouse model of hepatic fibrosis.

Gremlin, an antagonist of BMP-7, combines with BMP-7 and prevents BMP-7 binding to its own receptors, such as ALK3 and ALK6, resulting in the suppression of the BMP-7 signaling pathways (19). The role of gremlin in the regulation of bone formation during development is well recognized, however, of particular interest is the fact that the expression of gremlin is detectable in a range of human types of cancer (20). Additionally, expression of gremlin has been found to be induced by administration of TGF- $\beta$ in human tubuloepithelial cells (HK-2), also suggesting a role of gremlin in fibrogenesis (21). Taken together, specifying the expression pattern of gremlin in the progression of hepatic fibrosis and the crosstalk between BMP-7, gremlin, and TGF- $\beta$, will further expand our understanding of the mechanism underlying fibrogenesis, and will aid in the development of a novel biomarker for the detection of the severity of fibrosis.

\section{Materials and methods}

Animal grouping and treatment. Thirty male SPF C57BL/6 mice, weighing 22-25 g, were obtained from the Shanghai Laboratory Animal Center (Shanghai, China). All animals were maintained under specific pathogen-free conditions and had free access to standard laboratory water and chow. All 30 mice were randomly divided into three groups: the control group $(\mathrm{n}=6)$, the model group $(\mathrm{n}=18)$ and the BMP-7-treated group $(n=6)$. To understand the dynamic change of tissue factors during fibrogenesis, we further divided the model group into three subgroups according to different time-points: 6 weeks $(n=6), 8$ weeks $(n=6)$ and 12 weeks $(n=6)$. Hepatic fibrosis was induced in the model and the BMP-7-treated groups by hypodermic injection of carbon tetrachloride $\left(\mathrm{CCL}_{4}\right)$ (Sigma, USA) $0.5 \mathrm{ml} / 100 \mathrm{~g}$ body weight twice a week for 12 weeks. The mice in the control group were only injected with $0.2 \mathrm{ml}$ of physiological saline each time. The animals in the BMP-7-treated group were intraperitoneally administered human recombinant BMP-7 (Peprotech, USA), 300 pg/g each time, starting at 8 weeks after the first administration of $\mathrm{CCL}_{4}$ and lasting for 4 weeks, and were sacrificed at the end of the experiment. In the model group, mice were sacrificed at 6,8 and 12 weeks, respectively, and liver tissue and blood from each group were collected for further study. The above protocol for the animal experiment was approved by the institutional animal committee of Wenzhou Medical College.

Liver histological examination. Partial liver specimens were fixed in $10 \%$ neutral formalin and embedded in paraffin. The tissue sections were then cut into $4-\mu \mathrm{m}$ sections, and histological changes were assessed using H\&E and Masson's staining. Images from different groups were obtained using a video microscope.

Determination of serum chemical levels. Blood samples from each group were collected and the serum was isolated by $1,500 \mathrm{rpm}$ centrifugation for $5 \mathrm{~min}$. The serum samples were preserved to evaluate the levels of alanine transaminase (ALT), aspartate transaminase (AST), total bilirubin (Tbil) and albumin using an automatic biochemistry analyzer (Abbott Laboratories, USA), according to the manufacturer's protocol. Radioimmunoassay kit (Bei Jing Tigsun Diagnostics Co., Ltd., China) was used to detect the plasma levels of HA.

RNA isolation and reverse transcription-polymerase chain reaction $(R T-P C R)$. Total RNA was extracted from preserved liver tissue from each group using RNAiso Plus reagent (Aidlab Biotechnologies Co., China), according to the manufacturer's protocol. The RNA samples were then reverse-transcribed into cDNA by reverse transcription reaction which contained $2 \mu \mathrm{l}$ $\mathrm{MgCl}_{2}, 1 \mu 1$ 10X RT buffer, $3.75 \mu 1 \mathrm{R}$ Nase-free $\mathrm{dH}_{2} \mathrm{O}$, $1 \mu \mathrm{l}$ dNTP mixture, 0.25 RNase inhibitor, $0.5 \mu 1$ AMV reverse transcriptase, $0.5 \mu$ l Oligo dT-Adaptor primer and $1 \mu \mathrm{l}$ RNA sample. The above product was then used for amplification of cDNA, and the reaction system totalled $40 \mu \mathrm{l}$ volume per sample, which consisted of $10 \mu 1$ 5X PCR Buffer, $28.75 \mu 1$ DEPC-treated water, $0.25 \mu 1$ Takara Taq HS, $0.5 \mu 1$ upstream primers and $0.5 \mu \mathrm{l}$ downstream primers. The primers of the different cell cytokines used were as follows: BMP-7 (224 bp): sense primer, 5'-AACGCCACCAACCACGCCAT-3' and antisense primer, 5'-GGAAAGGTGTGGCCCCGCAA-3'. MMP-2 (252 bp): sense primer, 5'-TTGGGA CGTGGGACCCCGTT-3' and antisense primer, 5'-TGGGCGATGGTGCAGCGATG-3'. TIMP-2 (233 bp): sense primer, 5'-AGCCCTCCCTGAGCCGT GTT-3' and antisense primer, 5'-GAGCCAAACCGAGCCGT GCT-3'. $\alpha$-SMA (231 bp): sense primer, 5'-CCCTGCTCTGCC TCTAGCACACA-3' and antisense primer, 5'-TCCTGACCA CTAGAGGGGGCCA-3'. COL-I (204 bp): sense primer, 5'-GAGCGGAGAGTACTGGATCG-3' and antisense primer, 

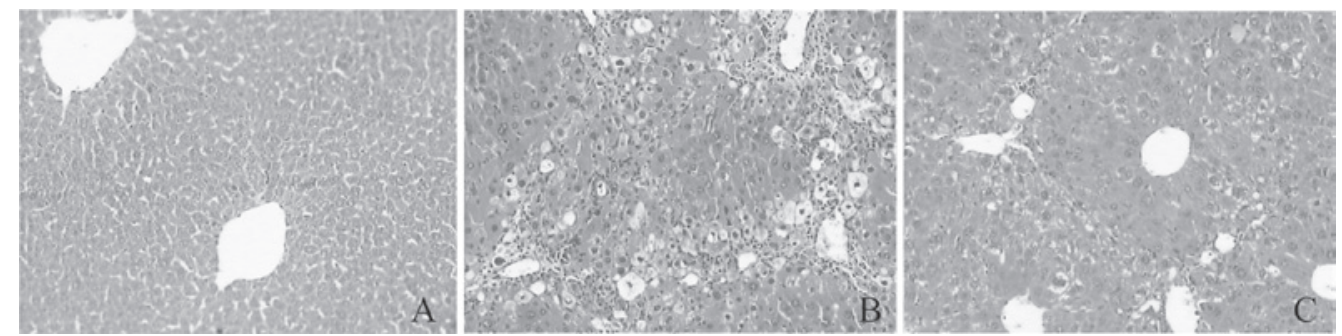

Figure 1. Histological assessment of liver sections by H\&E staining. (A) Liver sections from the control group; (B) Liver sections 12 weeks after repetitive injection of $\mathrm{CCL}_{4}$; (C) Liver sections from the BMP-7-treated group.
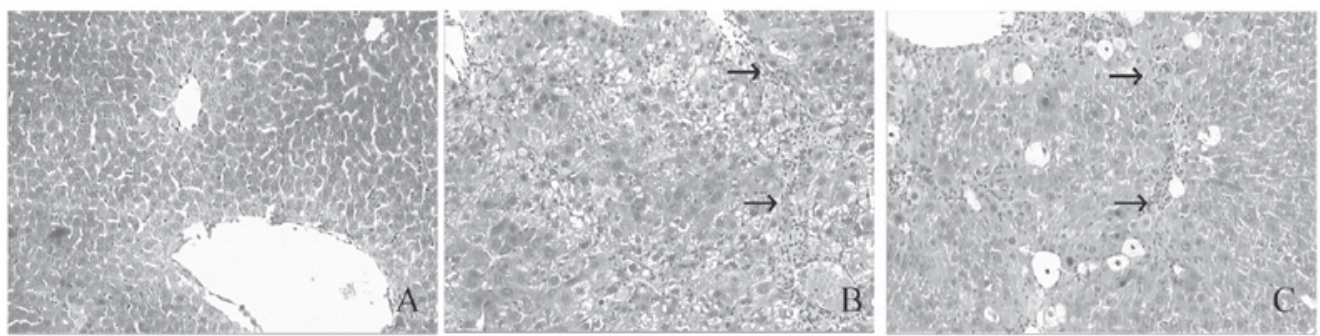

Figure 2. Masson's trichrome staining was used to detect the histological change in the liver sections from the different groups. (A) Control group; (B) 12-week subgroup; (C) BMP-7-treated group.

5'-TACTCGAATGGGAATCCAT-3'. TGF- $\beta 1$ (277 bp): sense primer, 5'-GCCCTCGGG AGCCACAAACC-3' and antisense primer, 5'-GCA GCAGGAGTCGCGGTGAG-3'. Gremlin (160 bp): sense primer, 5'-CGCAGCCGCACCATCCTCAA-3' and antisense primer, 5'-CGAGACCCGGGCATTCGAGC-3'. $\beta$-actin (452 bp): sense primer, 5'-GAGAGGGAAATCGTG CGTGAC-3' and antisense primer, 5'-CATCTGCTGGAA GGTGGACA-3'.

The PCR protocol was as follows: the mixture was first denatured at $94^{\circ} \mathrm{C}$ for $5 \mathrm{~min}, 35$ cycles at $94^{\circ} \mathrm{C}$ for $30 \mathrm{sec}$, $58^{\circ} \mathrm{C}$ for $30 \mathrm{sec}$ (TIMP-2), $60^{\circ} \mathrm{C}$ for $30 \mathrm{sec}$ (TGF- $\beta 1, \alpha$-SMA and gremlin), $61^{\circ} \mathrm{C}$ for $30 \mathrm{sec}\left(\mathrm{COL}-\mathrm{I}\right.$ and $\beta$-actin), $62^{\circ} \mathrm{C}$ for $30 \mathrm{sec}$ (MMP-2), $64^{\circ} \mathrm{C}$ for $30 \mathrm{sec}$ (BMP-7), followed by $72^{\circ} \mathrm{C}$ for $20 \mathrm{sec}$ and a final extension step at $72^{\circ} \mathrm{C}$ for $5 \mathrm{~min}$. The PCR products were finally analyzed in a $2 \%$ agarose gel containing ethidium bromide, and $\beta$-actin was used as a loading control.

Immunohistochemistry assay. Liver tissue specimens were fixed in neutral formalin, dehydrated in paraffin and then cut into $4-\mu \mathrm{m}$ sections, as described above. The sections were deparaffinized through xylene and rehydrated with decreasing graded alcohol. Subsequently, endogenous peroxidase activity was blocked by $3 \%$ hydrogen peroxide at $37^{\circ} \mathrm{C}$ for $15 \mathrm{~min}$, mixed with citrate buffer in a microwave oven for retrieving antigens and treated with primary antibody of gremlin (sc-28873, dilution 1:100) and TGF- $\beta 1$ (sc-52893, dilution 1:50) (Santa Cruz Biotechnology, Inc., USA) overnight at $4^{\circ} \mathrm{C}$, respectively. The samples were treated with a secondary antibody (PV-9001, PV-9002; Zhongshan Goldenbridge Biotechnology Co., Ltd., Beijing, China) at $37^{\circ} \mathrm{C}$ for $20 \mathrm{~min}$. The samples were first treated with DBA for $\sim 1$ min and then counterstained with hematoxylin for another $10 \mathrm{sec}$. PBS was used as a negative control for the primary antibody.
Protein isolation and western blotting. Total protein was isolated using lysis buffer (50 mM HEPES, $150 \mathrm{mM} \mathrm{NaCl}$, $1.5 \mathrm{mM} \mathrm{MgCl}_{2}, 5 \mathrm{mM}$ EDTA, $10 \%$ glycerol, $1 \%$ Triton X-100, $0.5 \%$ Nonidet P-40, $10 \mathrm{mM} \mathrm{NaF}$ and $1 \mathrm{mM} \mathrm{Na}_{3} \mathrm{VO}_{4} ; \mathrm{pH} 7.5$ ) supplemented with a protease inhibitor cocktail from Roche (Summerville, NJ, USA). Following heat denaturation at $95^{\circ} \mathrm{C}$ for $3 \mathrm{~min}$, the samples (15 $\mu \mathrm{g}$ protein each) were subjected to polyacrylamide gel electrophoresis (SDS-PAGE) and subsequently transferred onto a nitrocellulose membrane. The membrane was then blocked by skimmed milk for $1 \mathrm{~h}$ at room temperature. The primary antibodies against TGF- $\beta 1$ (sc-28873; Santa Cruz Biotechnology, Inc.), gremlin (sc-52893; Santa Cruz Biotechnology, Inc.) and GAPDH (SC-51907; Santa Cruz Biotechnology, Inc.) were used for incubation with the membrane overnight at $4^{\circ} \mathrm{C}$, respectively. After being washed with PBS three times, the membrane was incubated with the secondary antibody at room temperature for $1 \mathrm{~h}$, and then the film exposure was performed.

Statistical analysis. All values are expressed as the means \pm standard deviation (SD). Analysis of the statistical differences among multiple groups was determined by one-way analysis of variance (ANOVA) or the least significant difference (LSD) test. SPSS18.0 software was used for statistical analyses and $\mathrm{P}<0.05$ denoted a statistically significant difference.

\section{Results}

Effect of BMP-7 on hepatic fibrosis in $C C L_{4}$-treated mice. To better understand the detailed effect of BMP-7 on liver fibrosis, we established a mouse model of hepatic fibrosis by repetitive injection of $\mathrm{CCL}_{4}$ for 12 weeks. Human recombinant BMP-7 was administered during fibrogenesis. Liver samples from the control group showed normal hepatic cells and central 
Table I. Serum levels of ALT, AST, Alb and HA in the different experimental groups.

\begin{tabular}{lccccc}
\hline Group & $\mathrm{n}$ & ALT (U/l) & AST (U/l) & Alb (g/l) & HA (ng/ml) \\
\hline Control & 6 & $39.2 \pm 4.7$ & $41.9 \pm 5.3$ & $34.3 \pm 3.2$ & $117.8 \pm 11.2$ \\
Model & & & & \\
4-week subgroup & 6 & $90.8 \pm 7.2^{\mathrm{a}}$ & $112.2 \pm 9.2^{\mathrm{a}}$ & $30.9 \pm 0.4^{\mathrm{a}}$ & $363.9 \pm 31.9^{\mathrm{a}}$ \\
8-week subgroup & 6 & $158.5 \pm 11.6^{\mathrm{a}}$ & $173.3 \pm 21.4^{\mathrm{a}}$ & $26.8 \pm 0.4^{\mathrm{a}}$ & $497.5 \pm 58.4^{\mathrm{a}}$ \\
12-week subgroup & 6 & $191.3 \pm 24.5^{\mathrm{a}}$ & $206.6 \pm 25.0^{\mathrm{a}}$ & $22.2 \pm 1.2^{\mathrm{a}}$ & $548.2 \pm 29.1^{\mathrm{a}}$ \\
BMP-7-treated group & 6 & $153.9 \pm 18.1^{\mathrm{a}, \mathrm{b}}$ & $177.8 \pm 19.2^{\mathrm{a}, \mathrm{b}}$ & $25.4 \pm 0.9^{\mathrm{a}, \mathrm{b}}$ & $461.9 \pm 38.3^{\mathrm{a}, \mathrm{b}}$ \\
\hline
\end{tabular}

${ }^{\mathrm{a} C}$ Compared to the control group for $\mathrm{P}<0.05$; ${ }^{\mathrm{b}}$ compared to the 12 -week subgroup for $\mathrm{P}<0.05$. ALT, alanine transaminase; AST, aspartate transaminase; Alb, albumin; HA, hyaluronic acid.

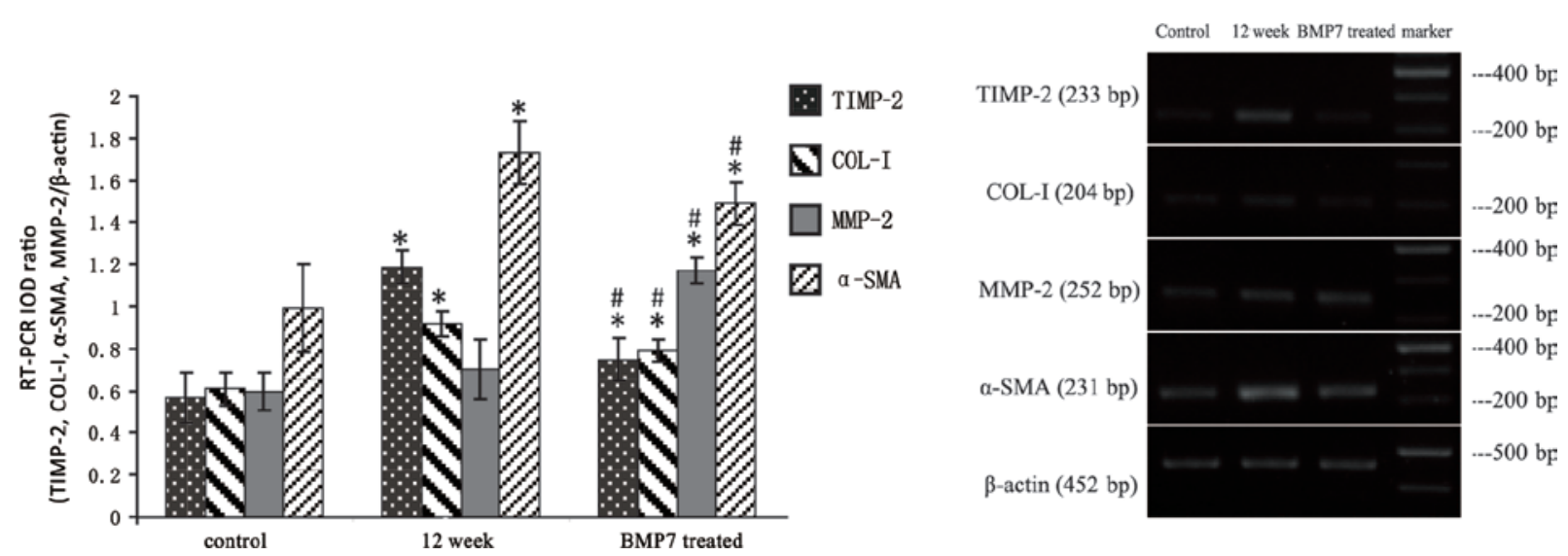

Figure 3. Gene expression patterns of TIMP-2, COL-I, MMP-2 and $\alpha$-SMA were evaluated by reverse transcription-PCR. Relative levels of mRNA were

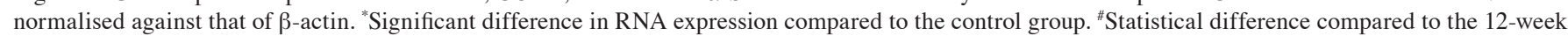
subgroup. $\mathrm{P}<0.05$.

veins by H\&E staining. By contrast, spotty hepatocellular necrosis and infiltration of inflammatory cells were evident in the model group (12 weeks), but were alleviated in the BMP-7-treated group (Fig. 1). Moreover, Masson's trichome staining presented with few collagen fibers in the normal liver tissue, whereas collagen in the model group was increased and enlarged, resulting in the formation of pseudo-lobules. Less collagenous fibers and reduced necrotic area were observed in the BMP-7-treated group, indicating a role of BMP-7 in the improvement of liver structure during fibrogenesis (Fig. 2).

The antifibrotic effect of BMP-7 was further demonstrated by the data from the serum markers of liver injury (Table I). The serum levels of ALT, AST as well as HA from the 12-week subgroup increased by approximately 5-fold, and a decreased level of ALB was also observed, as compared to the control group $(\mathrm{P}<0.05)$. However, BMP-7 significantly attenuated liver fibrosis based on the expression of serum markers. Significantly decreased ALT, AST and increased ALB levels were observed in the BMP-7-treated group compared to the 12-week subgroup, suggesting a role of BMP-7 in the improvement of liver function $(\mathrm{P}<0.05)$. Moreover, the fact that the severity of hepatic fibrosis was attenuated by the administration of BMP-7 was revealed by the downregulation of HA after the BMP-7 treatment, as compared to the 12-week subgroup $(\mathrm{P}<0.05)$. Taken together, our data indicate the potential anti- fibrotic effect of BMP-7 in a $\mathrm{CCL}_{4}$-treated mouse model of liver fibrosis.

Effect of BMP-7 on fibrosis-related genes in $\mathrm{CCL}_{4}$-treated mice. To further investigate the specific antifibrotic effect of BMP-7, the expression pattern of fibrosis-related cytokines was evaluated by RT-PCR. The mRNA levels of TIMP-2, COL-I, as well as $\alpha$-SMA, were upregulated in the progression of hepatic fibrosis, and a significant difference in levels was observed between the control and the 12-week subgroup $(\mathrm{P}<0.01$; Fig. 3). By contrast, the expression of the above cytokines was suppressed by the BMP-7 treatment, and the levels remained higher compared to the control group $(\mathrm{P}<0.01)$. However, the expression of MMP-2 was detectable in normal liver tissue, but there was no statistical difference between the control and the 12-week subgroup $(\mathrm{P}=0.192)$. Treatment of BMP-7 markedly increased the levels of MMP-2 compared to the control and the 12-week subgroup ( $\mathrm{P}<0.01$; Fig. 3).

Effect of BMP-7 on the expression of gremlin and TGF- $\beta 1$ in $\mathrm{CCL}_{4}$-treated mice. To clarify the effect of BMP-7 on the expression of gremlin and TGF- $\beta 1$, the dynamic changes of these cytokines were examined. Firstly, the mRNA level of BMP-7 was elevated gradually during fibrogenesis, which was downregulated by induction of BMP-7, suggesting a role 

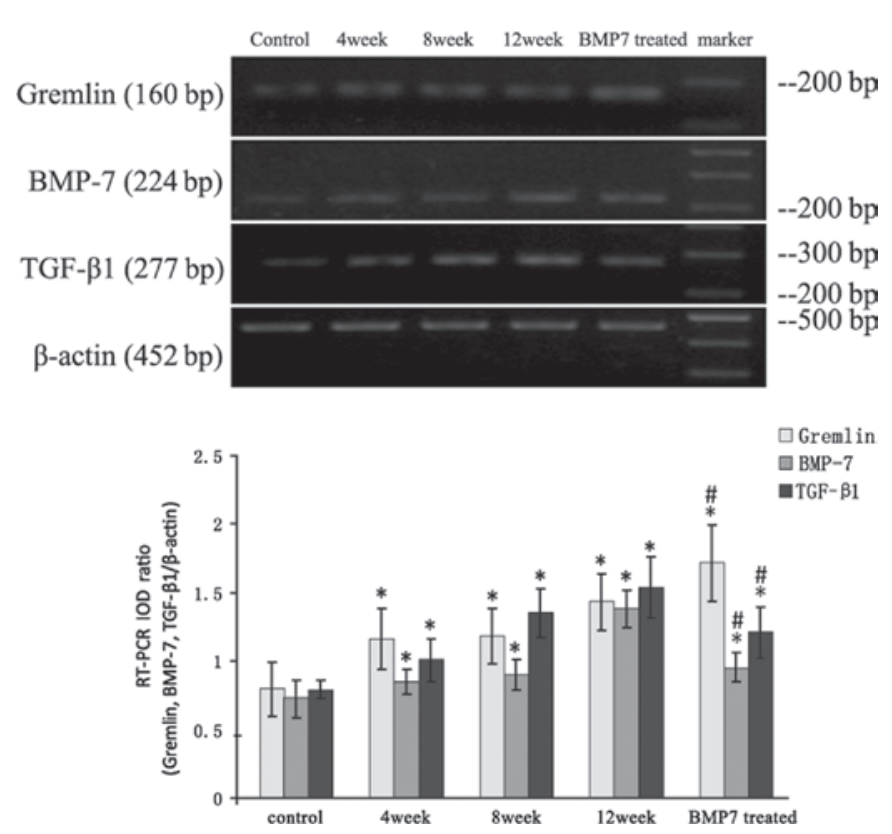

Figure 4. RNA levels of gremlin, TGF- $\beta 1$ and BMP-7 were detected by RT-PCR and normalized against those of $\beta$-actin. A statistical difference in gremlin, TGF- $\beta 1$ and BMP-7 was noted between the 12-week and the BMP7-treated group $(" \mathrm{P}<0.05)$. "Significant difference compared to the control group $(\mathrm{P}<0.05)$.

of BMP-7 in the inhibition of the endogenous expression of BMP-7 (P<0.05; Fig. 4).

Similarly, low mRNA expression of gremlin and TGF- $\beta 1$ was observed in normal liver tissues, which was gradually increased in the progression of hepatic fibrosis, and there was a statistically significant difference in each subgroup compared to the control group ( $\mathrm{P}<0.05 ;$ Fig. 4). Also, the mRNA level of TGF- $\beta 1$ was decreased in the BMP-7-treated group compared
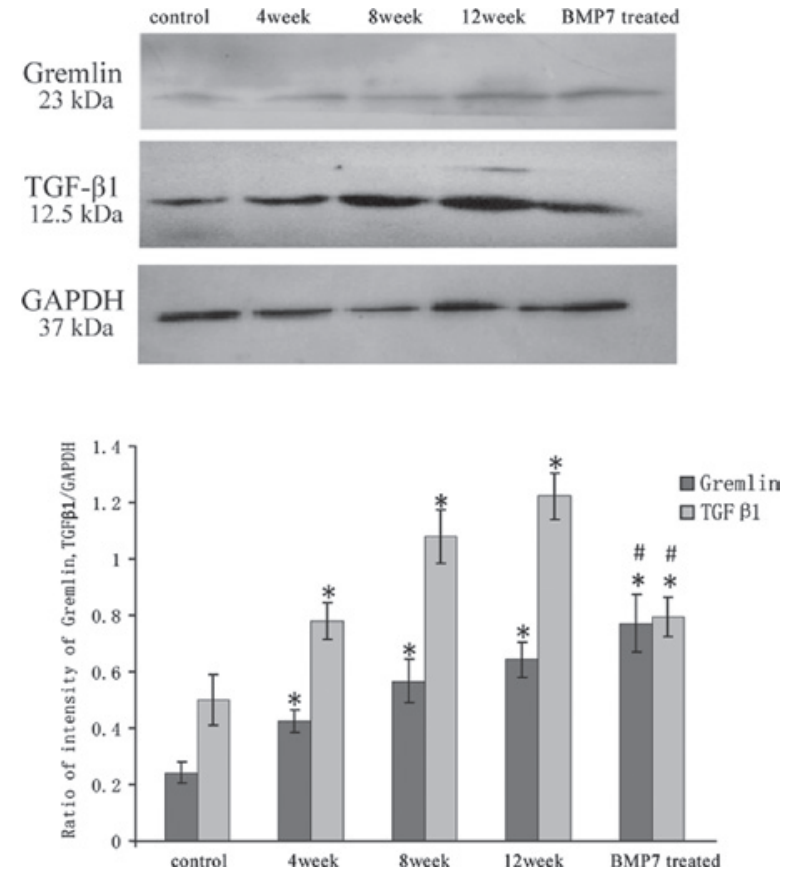

Figure 5. The protein levels of TGF- $\beta 1$ and gremlin were determined by western blotting and were further normalized to levels of GAPDH. $\mathrm{P}<0.05$.

to the 12-week subgroup $(\mathrm{P}<0.05)$. However, effects exerted by exogenous BMP-7 appeared to upregulate the expression of gremlin, which was different from that of TGF- $\beta 1$ and BMP-7 (Fig. 4). Additionally, the above expression pattern of gremlin and TGF- $\beta 1$ was also observed at the protein level, as demonstrated by either western blotting (Fig. 5) or immunohistochemistry (Figs. 6 and 7). Following immunostaining of liver sections from different groups, we found that gremlin and TGF- $\beta 1$ exhibited positive immunostaining mainly in hepatocytes, sinusoidal cells and fibroblasts of the periportal regions,
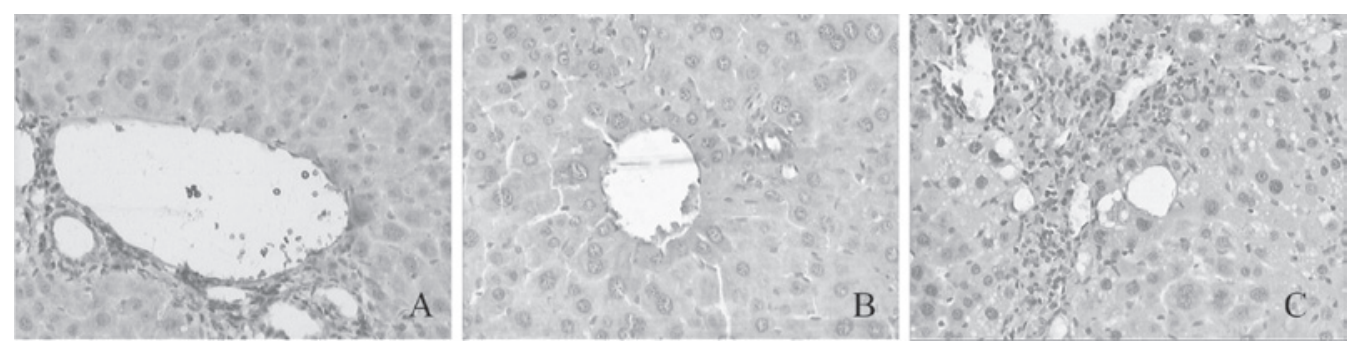

Figure 6. The localization and expression of gremlin were detected by immunostaining. (A) Control group; (B) 12-week subgroup; (C) BMP-7-treated group.
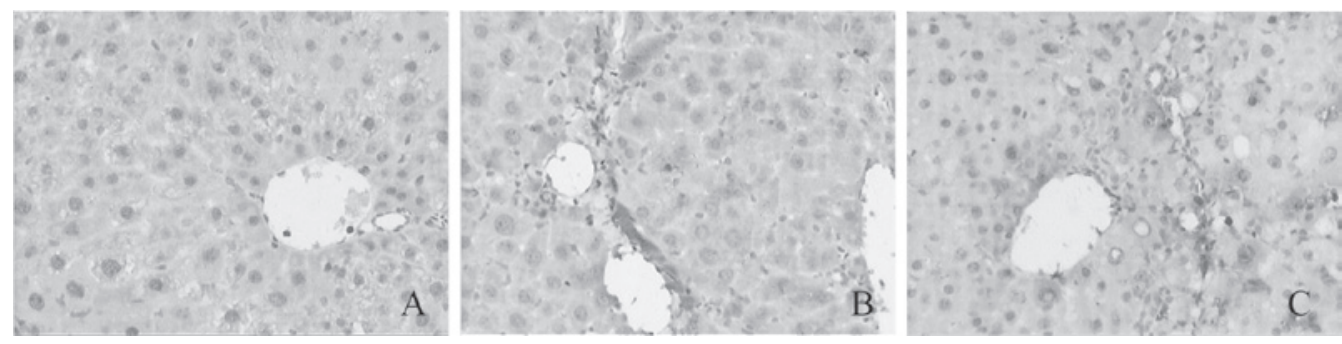

Figure 7. Immunostaining of TGF- $\beta 1$ in the liver sections of different groups. (A) Control group; (B) 12 -week subgroup; (C) BMP-7-treated group. 
suggesting a strong co-localization of gremlin and TGF- $\beta 1$ in fibrogenesis (Figs. 6 and 7).

\section{Discussion}

Liver fibrosis is the common response to chronic injury, a process with complex underlying integrated signaling networks and with various growth factors involved, of which the TGF- $\beta$ signaling pathway is crucial, and also considered as the most potent stimulator to HSCs (22). Among the TGF- $\beta$ subtypes, TGF- $\beta 1$ is revealed to closely correlate with the progression of fibrosis, that is in accordance with our data, whereby increased expression of TGF- $\beta 1$ was observed during the progression of hepatic fibrosis. On the other hand, BMP-7, belonging to the TGF- $\beta$ superfamily, has been well investigated since it plays an important role in organogenesis and development, whereas emerging published literature indicates that BMP-7 may be a new antagonist of organ fibrosis (23). However, whether BMP-7 is an antifibrotic mediator remains controversial. For example, Tacke et al showed that BMP-7 enhanced the expression of collagen in hTERT immortalized human HSCs, using an adenoviral vector-based BMP-7 expression system (24). To evaluate the antifibrotic effects of BMP7 in fibrosis, especially hepatic fibrosis, we established a mouse model induced by $\mathrm{CCL}_{4}$ and administered human recombinant BMP-7 in the process of fibrosis. Our results showed that BMP-7 suppressed the progression of liver fibrosis and improved liver function, partly via downregulation of COL-I, $\alpha$-SMA and TIMP-2, and upregulation of MMP-2. Although BMP-7 is mainly produced by the bone and kidney in vivo, we found low expression of BMP-7 in normal liver tissue, suggesting the involvement of the liver in the metabolism of BMP-7 in vivo.

Gremlin, a 184-amino acid protein, belongs to the cysteine knot family, and acts as an antagonist of BMP-7 by binding to BMP proteins. Due to the broad involvement of BMP-7 in the regulation of multiple cellular action, the abnormal expression of gremlin is widely correlated with pathological conditions. The highest expression of gremlin was found in samples from patients with idiopathic pulmonary fibrosis, suggesting a role of gremlin in fibrogenesis (25). Notably, Boers et al revealed the transcriptional profile of HSCs in three different statuses, including quiescence, half activated and fully activated (myofibroblasts), whereas the highly increased levels of gremlin were only observed in the latest stage of activated HSCs, not in the quiescence and half activated groups, indicating a potential biomarker of gremlin for the transdifferentiation of HSCs (26). Further studies by Boers et al revealed no expression of gremlin in normal mouse liver tissue, which was further evaluated by PCR. Accordingly, our data showed increased mRNA and protein levels of gremlin in the hepatic fibrosis model, whereas the expression of gremlin was detectable both in protein and mRNA levels in normal liver tissue, suggesting the involvement of gremlin not only in the progression of fibrosis, but also in the regulation of liver function in normal conditions. Thus, the expression pattern of gremlin in the progression of liver fibrosis may become another potential marker for assessing the severity of fibrosis, and the specific mechanisms of gremlin underlying fibrogenesis and the regulation of physiological conditions, require further investigation.
Our findings also show that the expressions of gremlin and TGF- $\beta 1$ are regulated by BMP-7. A strongly correlated expression pattern of gremlin and TGF- $\beta 1$ was observed in the progression of the hepatic fibrosis, but a difference was found after the administration of exogenous BMP-7. According to a previous study, the production of gremlin was stimulated by TGF- $\beta$ in the HK2 cell line, identifying gremlin as a downstream mediator of the TGF- $\beta$ signaling pathway (21). Thus, we suggest that the upregulated levels of gremlin induced by BMP-7 may be due to the increased expression of TGF- $\beta 1$ or the feedback effect of exogenous BMP-7.

In conclusion, we established a hepatic fibrosis mouse model by repetitive induction of $\mathrm{CCL}_{4}$. Improvement in the liver function and the regression of liver fibrosis were observed by in vivo delivery of BMP-7, due to the downregulation of fibrosis-related cytokines, such as COL-I, $\alpha$-SMA, as well as TIMP-2. Furthermore, the expression pattern of gremlin and TGF- $\beta 1$ during fibrogenesis and after treatment with BMP-7 was evaluated, providing new insights into the regulation of these factors by BMP-7 and offering novel biomarkers for the progression of hepatic fibrosis.

\section{Acknowledgements}

This study was supported by the Zhejiang Provincial Natural Science Foundation of China (Y207404).

\section{References}

1. Schuppan D and Afdhal NH: Liver cirrhosis. Lancet 371: 838-851, 2008.

2. Friedman SL: Evolving challenges in hepatic fibrosis. Nat Rev Gastroenterol Hepatol 7: 425-436, 2010.

3. Tsukada S, Parsons CJ and Rippe RA: Mechanisms of liver fibrosis. Clin Chim Acta 364: 33-60, 2006.

4. Friedman SL and Bansal MB: Reversal of hepatic fibrosis - fact or fantasy? Hepatology 43: S82-S88, 2006.

5. Mann DA and Mann J: Epigenetic regulation of hepatic stellate cell activation. J Gastroenterol Hepatol 23 (Suppl 1): 108-111, 2008.

6. Ghiassi-Nejad Z and Friedman SL: Advances in antifibrotic therapy. Expert Rev Gastroenterol Hepatol 2: 803-816, 2008.

7. Senoo H, Yoshikawa K, Morii M, Miura M, Imai K and Mezaki Y: Hepatic stellate cell (vitamin A-storing cell) and its relative - past, present and future. Cell Biol Int 34: 1247-1272, 2010.

8. Yu FJ, Dong PH, Fan XF, Lin Z, Chen YP and Li J: Downregulation of angiotensin II by shRNA reduces collagen synthesis in hepatic stellate cells. Int J Mol Med 25: 801-806, 2010.

9. Meyer C, Meindl-Beinker NM and Dooley S: TGF-beta signaling in alcohol induced hepatic injury. Front Biosci 15: 740-749, 2010.

10. Giannelli G, Mazzocca A, Fransvea E, Lahn M and Antonaci S: Inhibiting TGF-beta signaling in hepatocellular carcinoma. Biochim Biophys Acta 1815: 214-223, 2011.

11. De Bleser PJ, Niki T, Rogiers V and Geerts A: Transforming growth factor-beta gene expression in normal and fibrotic rat liver. J Hepatol 26: 886-893, 1997.

12. Inagaki Y and Okazaki I: Emerging insights into transforming growth factor beta Smad signal in hepatic fibrogenesis. Gut 56: 284-292, 2007.

13. Hong SW, Jung KH, Lee HS, et al: Suppression by fucoidan of liver fibrogenesis via the TGF-beta/Smad pathway in protecting against oxidative stress. Biosci Biotechnol Biochem 75: 833-840, 2011.

14. Sadick H, Herberger A, Riedel K, et al: TGF-betal antisense therapy modulates expression of matrix metalloproteinases in keloid-derived fibroblasts. Int J Mol Med 22: 55-60, 2008.

15. Soderberg SS, Karlsson G and Karlsson S: Complex and context dependent regulation of hematopoiesis by TGF-beta superfamily signaling. Ann NY Acad Sci 1176: 55-69, 2009. 
16. Massague J: How cells read TGF-beta signals. Nat Rev Mol Cell Biol 1: 169-178, 2000.

17. Derynck R and Zhang YE: Smad-dependent and Smadindependent pathways in TGF-beta family signalling. Nature 425: 577-584, 2003.

18. Zeisberg M, Hanai J, Sugimoto H, et al: BMP-7 counteracts TGF-beta1-induced epithelial-to-mesenchymal transition and reverses chronic renal injury. Nat Med 9: 964-968, 2003.

19. Zeisberg M and Kalluri R: Reversal of experimental renal fibrosis by BMP7 provides insights into novel therapeutic strategies for chronic kidney disease. Pediatr Nephrol 23: 1395-1398, 2008.

20. Claesson-Welsh L: gremlin: vexing VEGF receptor agonist. Blood 116: 3386-3387, 2010.

21. Carvajal G, Droguett A, Burgos ME, et al: gremlin: a novel mediator of epithelial mesenchymal transition and fibrosis in chronic allograft nephropathy. Transplant Proc 40: 734-739, 2008.

22. Shen H, Huang G, Hadi M, et al: Transforming growth factorbeta1 downregulation of Smad1 gene expression in rat hepatic stellate cells. Am J Physiol Gastrointest Liver Physiol 285: G539-G546, 2003.
23. Weiskirchen R, Meurer SK, Gressner OA, Herrmann J, Borkham-Kamphorst E and Gressner AM: BMP-7 as antagonist of organ fibrosis. Front Biosci 14: 4992-5012, 2009.

24. Tacke F, Gabele E, Bataille F, et al: Bone morphogenetic protein 7 is elevated in patients with chronic liver disease and exerts fibrogenic effects on human hepatic stellate cells. Dig Dis Sci 52: 3404-3415, 2007.

25. Koli K, Myllarniemi M, Vuorinen K, et al: Bone morphogenetic protein-4 inhibitor gremlin is overexpressed in idiopathic pulmonary fibrosis. Am J Pathol 169: 61-71, 2006.

26. Boers W, Aarrass S, Linthorst C, Pinzani M, Elferink RO and Bosma P: Transcriptional profiling reveals novel markers of liver fibrogenesis: gremlin and insulin-like growth factor-binding proteins. J Biol Chem 281: 16289-16295, 2006. 